\title{
Correction to: Guidance to 2018 good practice: ARIA digitally-enabled, integrated, person-centred care for rhinitis and asthma
}

\author{
J. Bousquet ${ }^{1,2,3,4^{*}}$ (I) and The MASK study group
}

\section{Correction to: Clin Transl Allergy (2019) 9:16 https://doi.org/10.1186/s13601-019-0252-0}

Following publication of the original article [1], the authors reported that one of the collaborators' names was spelled incorrectly. In this Correction the incorrect and correct author name are shown. In the author list of this Correction article, only the corresponding author and institutional author are presented.

Originally the collaborator name was published as:

- M. van Hague

The correct collaborator name is:

- M. van Hage

\section{Author details}

${ }^{1}$ MACVIA-France, Fondation Partenariale FMC VIA-LR, CHU Arnaud de Villeneuve, 371 Avenue du Doyen Gaston Giraud, 34295 Montpellier Cedex 5, France. ${ }^{2}$ INSERM U 1168, VIMA: Ageing and Chronic Diseases Epidemiological and Public Health Approaches, Villejuif, Université Versailles St-Quentin-enYvelines, UMR-S 1168, Montigny Le Bretonneux, France. ${ }^{3}$ Euforea, Brussels, Belgium. ${ }^{4}$ Humboldt-Universität zu Berlin, Berlin Institute of Health, Comprehensive Allergy Center, Department of Dermatology and Allergy, Charité, Universitätsmedizin Berlin, Berlin, Germany.

The original article can be found online at https://doi.org/10.1186/s1360 1-019-0252-0.

Published online: 09 October 2019

\section{Reference}

1. Bousquet J, Bedbrook A, Czarlewski W, Onorato GL, Arnavielhe S, Laune D, Mathieu-Dupas E, Fonseca J, Costa E, Lourenço O, Morais-Almeida M, Todo-Bom A, Illario M, Menditto E, Canonica GW, Cecchi L, Monti R, Napoli L, Ventura MT, De Feo G, Fokkens WJ, Chavannes NH, Reitsma S, Cruz AA, da Silva J, Serpa FS, Larenas-Linnemann D, Fuentes Perez JM, Huerta-Villalobos YR, Rivero-Yeverino D, Rodriguez-Zagal E, Valiulis A, Dubakiene R, Emuzyte R, Kvedariene V, Annesi-Maesano I, Blain H, Bonniaud P, Bosse I, Dauvilliers Y, Devillier P, Fontaine JF, Pépin JL, Pham-Thi N, Portejoie F, Picard R, Roche N, Rolland C, Schmidt-Grendelmeier P, Kuna P, Samolinski B, Anto JM, Cardona V, Mullol J, Pinnock H, Ryan D, Sheikh A, Walker S, Williams S, Becker S, Klimek L, Pfaar O, Bergmann KC, Mösges R, Zuberbier T, Roller-Wirnsberger RE, Tomazic PV, Haahtela T, Salimäki J, Toppila-Salmi S, Valovirta E, Vasankari T, Gemicioğlu B, Yorgancioglu A, Papadopoulos NG, Prokopakis EP, Tsiligianni IG, Bosnic-Anticevich S, O'Hehir R, Ivancevich JC, Neffen H, Zernotti ME, Kull I, Melén E, Wickman M, Bachert C, Hellings PW, Brusselle G, Palkonen S, Bindslev-Jensen C, Eller E, Waserman S, Boulet LP, Bouchard J, Chu DK, Schünemann HJ, Sova M, De Vries G, van Eerd M, Agache I, Ansotegui IJ, Bewick M, Casale T, Dykewick M, Ebisawa M, Murray R, Naclerio R, Okamoto Y, Wallace DV, The MASK study group. Guidance to 2018 good practice: ARIA digitally-enabled, integrated, person-centred care for rhinitis and asthma. Clin Transl Allergy. 2019;9:16. https://doi.org/10.1186/s13601-019-0252-0.

\section{Publisher's Note}

Springer Nature remains neutral with regard to jurisdictional claims in published maps and institutional affiliations.

*Correspondence: jean.bousquet@orange.fr

${ }^{1}$ MACVIA-France, Fondation Partenariale FMC VIA-LR, CHU Arnaud de Villeneuve, 371 Avenue du Doyen Gaston Giraud, 34295 Montpellier

Cedex 5, France

Full list of author information is available at the end of the article 